\title{
The problem of fastening cargo on railway rolling stock
}

\author{
Khabibulla Turanov ${ }^{1 *}$ and Yadgor Ruzmetov ${ }^{2}$ \\ ${ }^{1}$ Tashkent state technical University named Islam Karimov, University Str., 2, 100174. Tashkent \\ Uzbekistan \\ ${ }^{2}$ Tashkent Railway Engineering Institute, Temirylchilar Str., 1, 100167. Tashkent, Uzbekistan
}

\begin{abstract}
Fastening of cargo on the car. Calculation of load shift, elongation and forces in elastic elements of fasteners. Describe the parameters of the load and the physical and geometric parameters of the load fastening; give the results of the calculation of the longitudinal forces perceived by the elements of the load fastening. The article uses the basic law of dynamics in relative motion for the non-ideal connection known from theoretical mechanics. The results of the calculation of the longitudinal forces perceived by the load fastening elements, which allowed determining the load shifts along the car, elongation and forces in the flexible fastening elements are presented. Example calculation contributes to the practical application of a new method of calculating the elements of fastening on the car in the development of schemes of placement and fastening of goods of arbitrary geometry. The results of calculations revealed that the accepted value of the coefficient of longitudinal dynamics of the car corresponds to the shunting collision of the car on the hump yard in the marshalling yards.
\end{abstract}

\section{Introduction}

This paper, as well as works [1 - 22], is devoted to problems of fastening of freights on the open rolling stock. It should be noted that in [17] does not define shifts of cargo along the car, elongation and efforts in flexible fastening elements, which confirm the incorrectness of the solution of the problem of cargo fastening. It is for this reason that in [17] the calculated values of the forces in the fastenings of the goods, both in the longitudinal and transverse directions, regardless of the shape of the cargo and the useful floor space of the car, have the same value, which is not true. In $[12,16,18]$ new methods of calculation of elastic elements of fastening of the load at influence both longitudinal, and transverse forces are stated. Specific examples of calculations against longitudinal shear using new methods are given in [18].

*Corresponding author: khturanov@yandex.ru 
However, up to date, there are few examples of the application of engineering calculation of the load shift along the car, elongation and effort in the flexible fastening elements by determining the number of fasteners, which would provide guaranteed strength of flexible fastening elements according to the new method [12,16, 18], which confirms the actual technical problem of securing goods on a railway platform.

\section{Object:}

- describe the parameters of the cargo and physical and geometric parameters of cargo securing;

- give results of calculation of the longitudinal forces perceived by elements of cargo fastening;

- describe the sequence of calculation of forces in the elastic fastening elements according to the recommended formulas.

\section{Method of research}

The research methods are based on the use of the basic law of dynamics in relative motion for non-ideal coupling, known from theoretical mechanics [12].

\section{The main results of the study}

For example, we show the results of calculations of forces in the flexible elements of fastening the winding gear LP-152 on the railway platform (Fig. 1, Fig.2, Fig.3).

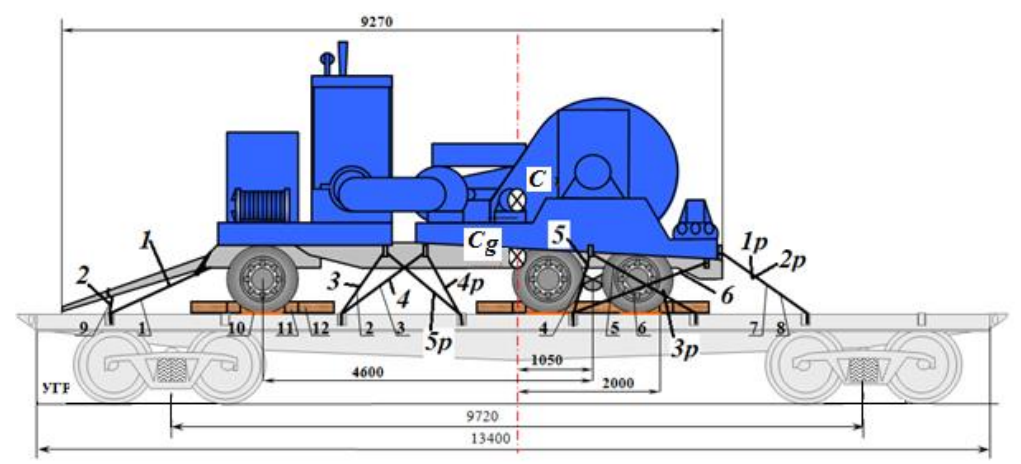

Fig. 1. Real scheme of placement and fastening of cargo on the car together with elastic and resistant means of fastening- the main view.

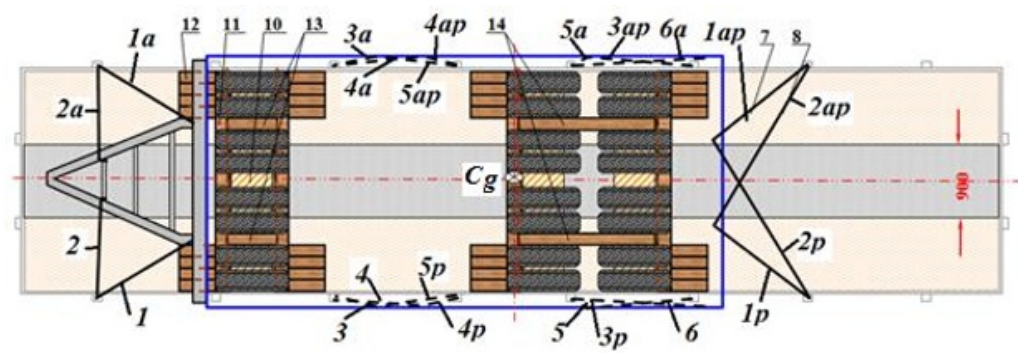

Fig. 2. Real scheme of placement and fastening of cargo on the car together with elastic and resistant means of fastening- top view. 


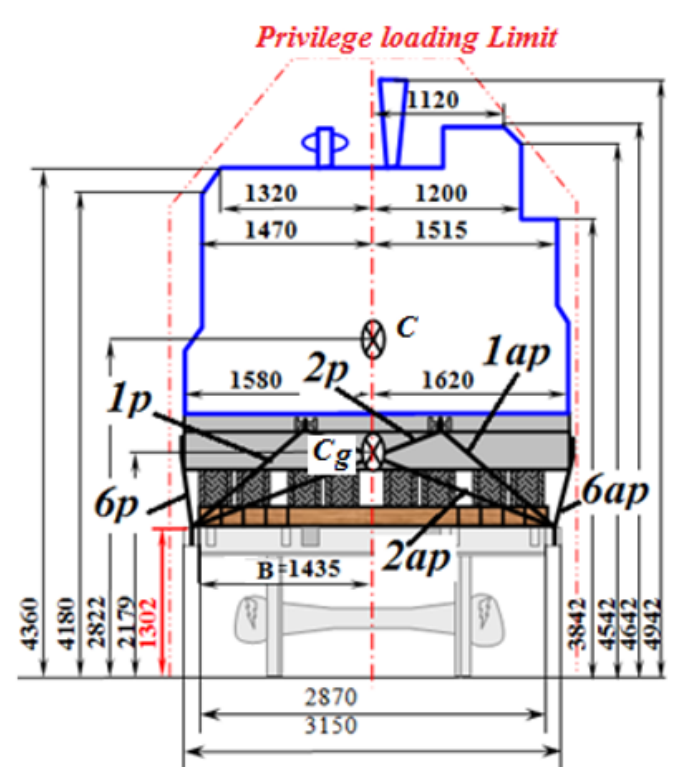

Fig. 3. Real scheme of placement and fastening of cargo on the car together with elastic and resistant means of fastening - side view.

In Fig. 1 the numbers indicate flexible fastening elements; $R_{1}-R_{6}, R_{1 a}-R_{6 a}$ - elastic fastening elements in one direction; $R_{1 p}-R_{5 p}, R_{1 a p}-R_{5 a p}$ - the other direction.

Loading is designed on a 4-axis universal railway platform with a wooden or woodmetal floor with a strip width of not more than $1100 \mathrm{~mm}$. Cargo is installed on the floor of the platform without shifting the center of gravity from the transverse axis of the platform.

The longitudinal and transverse forces on the cargo during transport are perceived as:

- in the longitudinal direction by six pairs of extensions $R 1-R 6$ (2 pairs of extensions from a wire with a diameter of $6 \mathrm{~mm}$ in 8 threads in each direction) and blocks of the position No. 10, 11, 12;

- in the transverse direction of five stretch marks $R_{1}-R_{5}$ и $R_{1 p}-R_{5 p}$ in each of the directions and blocks of the position No. 13 and 14 are nailed to the floor platform 6 nails K6x 200 (mm) each frame.

\section{Determination of physical and geometrical parameters of cargo securing}

1. Parameters of flexible fastening elements (set values and accepted from the drawing of the load).

1.1. Physical and geometric parameters of elastic fastening elements, as in $[12,18]$ :

$E=1 \cdot 10^{7}-$ the modulus of elasticity of the elastic fastening elements taking into account the twisting of the wire $\left(\mathrm{kN} / \mathrm{m}^{2}\right) ; d=6-$ is the wire diameter of the elastic fixing element, $\mathrm{mm} ; n-$ is the number of strands of wire elastic fixing element (for stretch marks and bindings $n=8)(\mathrm{PC}) ; R 0=20$ - is adopted the average value of the effort of preliminary wire twists of fastenings $(\mathrm{kN})$.

Let us make a reservation that at neglect of physical and geometrical parameters of elastic elements of fastening it is not possible to make any calculations of shift of the load along the car $[12,18]$.

Table 1 summarizes these geometric parameters of the securing devices. 
Table 1. Geometry of securing devices (m).

\begin{tabular}{|c|c|c|c|c|c|c|c|}
\hline $\begin{array}{l}\text { Designation } \\
\text { of the } \\
\text { securing }\end{array}$ & $\begin{array}{l}\text { Direction of } \\
\text { securing }\end{array}$ & The valu & the pr & ons of tl & curing $\mathrm{c}$ & es and $\mathrm{t}$ & ngth (m) \\
\hline$a_{i}$ & \multirow{6}{*}{ One direction } & 1.19 & 0.041 & 0.52 & 1.095 & 0.232 & 1.861 \\
\hline$h_{i}$ & & 0.605 & 0.302 & 0.907 & 0.907 & 0.907 & 0.907 \\
\hline$l_{i}$ & & 1.467 & 1.087 & 1.06 & 1.432 & 0.952 & 12.078 \\
\hline$a_{i p}$ & & 1.19 & 1.286 & 1.382 & 0.52 & 0.903 & 0 \\
\hline$h_{i p}$ & & 1.008 & 1.008 & 0.907 & 0.907 & 0.907 & 0 \\
\hline$l_{i p}$ & & 1.786 & 2.515 & 1.662 & 1.06 & 1.292 & 0 \\
\hline$a_{i a}$ & \multirow{6}{*}{$\begin{array}{l}\text { The other } \\
\text { direction }\end{array}$} & 1.19 & 0.041 & 0.52 & 1.095 & 0.232 & 1.861 \\
\hline$h_{i a}$ & & 0.605 & 0.302 & 0.907 & 0.907 & 0.907 & 0.907 \\
\hline$l_{i a}$ & & 1.497 & 1.087 & 1.06 & 1.432 & 0.952 & 2.078 \\
\hline$a_{\text {iap }}$ & & 1.286 & 1.286 & 1.382 & 0.52 & 0.903 & 0 \\
\hline$h_{i a p}$ & & 1.008 & 1.008 & 0.907 & 0.907 & 0.907 & 0 \\
\hline$l_{\text {iap }}$ & & 1.851 & 2.515 & 1.662 & 1.06 & 1.292 & 0 \\
\hline
\end{tabular}

\section{Determination of longitudinal forces.}

1. The longitudinal portable force of inertia $I_{e x}[12,18]$.

$a_{e x}=1,6 \mathrm{~g}=15.696-$ longitudinal portable acceleration of the $\mathrm{car}\left(\mathrm{m} / \mathrm{s}^{2}\right)$;

$k_{d . x}=a_{e x} / \mathrm{g}=1,6$ - factor longitudinal dynamics of the carriage $(1,2 \div 2)$ (portable longitudinal acceleration of the car with a load in the units of $\mathrm{g}$ );

$I_{e x}=k_{d . x} G=1,6 \cdot 450=720$ - longitudinal portable force of inertia $(\mathrm{kN})$.

2. Friction force [18].

$F_{f r . x}=f \cdot G=0.315 \cdot 450=141.75-$ sliding friction force $(\mathrm{kN})($ where $f=0.315-$ sliding friction coefficient "metal-wood").

3. Longitudinal shear force [19] $(\mathrm{kN})$ :

$F_{\text {long. }}=I_{e x}=720$ или $F_{\text {shear } . x}=F_{x}=F_{\text {long. }}=720$.

4. Determination of the longitudinal force perceived by the elastic fastening elements of the cargo when considering only the force of friction of the cargo is the friction force of clutch like in $[12,16])(\mathrm{kN})$ :

$\Delta F_{\text {long. }}=\Delta F_{x}=F_{\text {shear. } x}-F_{\text {fr. } x}=720-141.75=578.25$.

\section{Calculation of the force in the elastic fastening elements according to the recommended formulas}

1. The definition of the projection elastic force of the mounting longitudinal axis when considering the efforts of preliminary twisting of fastening wire $R 0$ [18].

1.1. Calculation of the projection of elastic forces from the force of preliminary twists of the fastening wire of only one direction according: 


$$
R 0_{x 1}=R 0 \times\left(\frac{a_{1}}{l_{1}}+\frac{a_{1 a}}{l_{1 a}}+\frac{a_{2}}{l_{2}}+\frac{a_{2 a}}{l_{2 a}}+\ldots\right) .
$$

Substituting in the last formula the initial data and taking into account the symmetrical placement of the load relative to the longitudinal axis of the car [18], we obtain $(\mathrm{kN})$

$$
\begin{aligned}
& R 0_{x 1}=20 \times 2 \times\left(\frac{1.19}{1.467}+\frac{0.041}{1.087}+\frac{0.52}{1.06}+\frac{1.095}{1.432}+\frac{0.232}{0.952}+\frac{1.861}{2.078}\right)= \\
& =40 \times(0.811+0.038+0.49+0.769+0.244+0.896)=40 \times 3.24=129.73 ;
\end{aligned}
$$

FROreat. $x 1=R 0_{x 1}=129.7$ - holding power in fastening elements from the efforts of preliminary twisting of the wire in one direction $(\mathrm{kN})$.

1.2. Calculation of the projection of elastic forces from the force of preliminary twists of the wire fastening of the other direction:

$$
R 0_{x 2}=R 0 \times\left(\frac{a_{1 p}}{l_{1 p}}+\frac{a_{1 a p}}{l_{1 a p}}+\frac{a_{2 p}}{l_{2 p}}+\frac{a_{2 a p}}{l_{2 a p}}\right) .
$$

Substituting in the last formula initial data and considering symmetric placement of freight concerning a longitudinal axis of the car, we have

FROreat. $x 2=R 0_{x 2}=128.52$ - holding power in fastening elements from the efforts of preliminary twisting of the wire in the other direction $(\mathrm{kN})$.

2. Determination of the projection of the force (elastic forces) of the preliminary twists of the fastening wire $R 0$ on the vertical axis [18].

2.1. Calculation of the projection of the force of preliminary twists of the wire fastening of one direction

$$
R 0_{z 1}=R 0 \times\left(\frac{h_{1}}{l_{1}}+\frac{h_{1 a}}{l_{1 a}}+\frac{h_{2}}{l_{2}}+\frac{h_{2 a}}{l_{2 a}}+\frac{h_{3}}{l_{3}}+\frac{h_{3 a}}{l_{3 a}}+\frac{h_{4}}{l_{4}}+\frac{h_{4 a}}{l_{4 a}}+\ldots\right) .
$$

Substituting in the last formula the initial data and taking into account the symmetrical placement of the load relative to the longitudinal axis of the car [18], we have $(\mathrm{kN})$

$$
\begin{aligned}
& R 0_{z 1}=20 \times 2 \times\left(\frac{0.605}{1.467}+\frac{0.302}{1.087}+\frac{0.907}{1.06}+\frac{0.907}{1.432}+\frac{0.907}{0.952}+\frac{0.907}{2.078}\right)= \\
& =40 \times(0.412+0.278+0.858+0.633+0.952+0.432)=40 \times 3.569=142,76 .
\end{aligned}
$$

$R 0 z 1 x=R 0 z 1=142.76$.

2.2. Calculation of the projection of the force of preliminary twists of the fastening wire on the vertical axis of the other direction

$$
R 0_{z 2}=R 0 \times\left(\frac{h_{1 p}}{l_{1 p}}+\frac{h_{1 a p}}{l_{1 a p}}+\frac{h_{2 p}}{l_{2 p}}+\frac{h_{2 a p}}{l_{2 a p}}+\frac{h_{3 p}}{l_{3 p}}+\frac{h_{3 a p}}{l_{3 a p}}+\frac{h_{4 p}}{l_{4 p}}+\frac{h_{4 a p}}{l_{4 a p}}+\ldots\right) .
$$

Substituting in the last formula the initial data and taking into account the symmetrical placement of the load relative to the longitudinal axis of the car, we will have:

$R 0 z 2 x=R 0 z 1 x=R 0 z 1=122.37$ - projections of elastic forces from preliminary wire twist of fastenings on the vertical axis the other direction $(\mathrm{kN})$.

3. Determination of the sliding friction force from the force of preliminary twists of the fastening wire $R 0$ on the vertical axis $[12,18]$.

Calculation of sliding friction force:

$$
F R 0_{f r . x}=f \times \sum_{i=1}^{n_{\mathrm{p}}} R 0_{i z}
$$


$F R 0_{\text {fr } x . x 1}=f \times R 0_{z 1 x}=0.315 \times 142.76=45.0-$ sliding friction force from the pre-twisting of wire for mounting in one direction $(\mathrm{kN})$;

$F R 0_{\text {fr. } x 2}=f \times R 0_{i z 2 x}=0.315 \times 122.37=38.55$ - the sliding friction force from the preliminary twists of the fastening wire due to the symmetry of the other direction $(\mathrm{kN})$.

Thus, the results of calculations, as in [8], proved that the projection of the load securing elements on the vertical axis affect the magnitude of the friction force along the longitudinal axis of the car. This increases the value of the holding forces.

4. Determination of the reaction of thrust blocks (see (4.5) [12]).

$\left[R_{\text {nail }}\right]=1,08-$ permissible value of force on one fastener (nail) $(\mathrm{kN})[1,2,16]$;

$n_{\text {nail } x}=16-$ the accepted quantity of thrust blocks according to the scheme of placement and fastening of freight on the car, pieces (see Fig. 1);

$k_{1}=0.6$ is the coefficient of strength of attachment resistant bricks, taking into account the condition of the floor of the car, pieces (usually takes $0.5 \div 0.6$ );

$n_{\text {bar.x }}=6-$ the accepted value of the required number of nails for each thrust block (PCs.);

$R_{\text {bar. } x}=k_{1} n_{\text {nail. } x} n_{\text {bar. } x}\left[R_{\mathrm{\kappa p}}\right]=0.6 \cdot 16 \cdot 6 \cdot 1.08=62.2-$ reaction of thrust blocks with the accepted number of nails (kN) (also see (4.5) in [12]).

5. Determination of the sum of all "holding" forces along the car [18]:

$F R 0_{x 1}=F_{f r . x}+F R 0_{x 1}+F R 0_{\text {тp } x 1}=141.75+129.73+45.0=316.5-$ "holding" forces without taking into account the reaction of thrust blocks of one direction $(\mathrm{kN})$;

$\Delta F R 0_{\text {ret. } x 1}=F R 0_{x 1}+R_{\text {bar. } x}=316.48+62.2=378.7-$ "holding" forces taking into account reaction of thrust blocks of one direction $(\mathrm{kN})$;

$F R 0_{x 2}=F_{\text {fr. } x}+F R 0_{x 2}+F R 0_{\text {fr. } x 2}=141.75+128.52+38.55=308.8-$ "holding" power without taking into account the reaction of the thrust blocks other direction, in $(\mathrm{kN})$;

$\Delta F R 0_{\text {ret. } x 2}=F R 0_{x 2}+R_{\text {bar } x}=308.8+62.2=371.0-$ "holding" forces taking into account reaction of thrust blocks of other direction $(\mathrm{kN})$.

6. Longitudinal force perceived by fastening elements of the cargo $(\mathrm{kN})$ :

$\Delta F R 0_{x 1}=F_{\text {shear } x}-F R 0_{\text {ret } x 1}=720-378.7=341.3 \mathrm{kN}$ (see (14) [16]) - the difference between shearing and restraint forces in one direction;

$\Delta F R 0_{x 2}=F_{\text {shear } x}-F R 0_{\text {ret. } x 2}=720-371.0=349.0 \mathrm{kN}-$ the difference between shearing and restraining forces of another direction.

The positive sign of the longitudinal force $\triangle F R 0 x$ means that the shear force is greater than the holding force. In this case, obviously there will be a shift of the cargo along the car, which is accompanied by loss of fasteners (nail) resistant bars and twisting these bars are relatively distant from the end surfaces of the load faces.

7. Calculation of equivalent rigidity of fastening elements along the car $c_{\text {equiv. } x}$ at the coefficient of sliding friction (after the cargo shift occurs).

7.1. Calculation due to the asymmetry of the fastening elements relative to the transverse axis of one direction (see (3.90 a) [12] or (20) [16]):

$$
c_{\text {equiv. } x}=7,854 \times d_{i}^{2} \times \sum_{i=1}^{n_{\mathrm{p}}} \frac{n_{i}}{l_{i}} \times\left(f \times \frac{h_{i}}{l_{i}}+\frac{a_{i}}{l_{i}}\right) \times \frac{a_{i}}{l_{i}},
$$

or when $\mathrm{f}=0.315$, di $=6$ (mm), ni $=8$ (PCs.): 


$$
c_{\text {equiv. } x 1}=7,854 \times 6^{2} \times 2 \times\left[\begin{array}{l}
\frac{8}{1.467} \times\left(0.315 \times \frac{0.605}{1.467}+\frac{1.19}{1.467}\right) \times \frac{1.19}{1.467}+ \\
+\frac{8}{1.087} \times\left(0.315 \times \frac{0.302}{1.087}+\frac{0.041}{1.087}\right) \times \frac{0.041}{1.087}+ \\
+\frac{8}{1.06} \times\left(0.315 \times \frac{0.907}{1.06}+\frac{0.52}{1.06}\right) \times \frac{0.52}{1.06}+ \\
+\frac{8}{1.432} \times\left(0.315 \times \frac{0.907}{1.432}+\frac{1.095}{1.432}\right) \times \frac{1.095}{1.432}+ \\
+\frac{8}{0.952} \times\left(0.315 \times \frac{0.907}{0.952}+\frac{0.232}{0.952}\right) \times \frac{0.232}{0.952}+ \\
+\frac{8}{2.078} \times\left(0.315 \times \frac{0.907}{2.078}+\frac{1.861}{2.078}\right) \times \frac{1.86195}{2.078}
\end{array}\right]
$$

or, after calculations,

$$
c_{\text {equiv } . x 1}=565.5 \times 31.68=8937 .
$$

Finally get: $c_{\text {equiv.x }}=8937(\mathrm{kN} / \mathrm{m})$.

7.2. Due to the asymmetry of the fastening elements relative to the longitudinal axis of the other direction, calculating the equivalent stiffness of the fastening elements along the car $c_{\text {equiv.x }}$, analogous to $c_{\text {equiv.x }}$, finally we get: $c_{\text {equiv. } x 2}=565.5 \cdot 28.824=8150(\mathrm{kN} / \mathrm{m})$.

8. Calculation of the load shift along the car.

$\Delta F R 0 x=341.3 \mathrm{kN}-$ shear strength, perceived means of securing cargo (see p. 6); $\Delta x 0=$ 0,006 - the initial shift of cargo in conjunction with thrust blocks, $m[12,18]$.

8.1. Calculation of load shear for one direction [19]

$$
\Delta x=\Delta F R 0 x / c_{\text {equiv. } x}+\Delta x_{0} .
$$

$\Delta x 1=\Delta F R 0 x 1 / c_{\text {equiv. } x 1}+\Delta x 0=341.3 / 8987+0.006=0.038+0.006=0.044-$ load shift in one direction $(\mathrm{m})(44 \mathrm{~mm})$;

8.2. Calculation of load shift for another direction $[12,18]$

$\Delta x 2=\Delta F R 0 \times 2 / c_{\text {equiv. } x 2}+\Delta x 0=349.0 / 8150+0.006=0.043+0.006=0.049-$ load shift in the other direction $(\mathrm{m})(49 \mathrm{~mm})$.

9. Calculation of the elongation and force of each flexible fastening element under the influence of longitudinal forces.

9.1. Calculation of the elongation of each flexible fastening element corresponding to the shift of the load along the car (see $(3.80 \mathrm{~b})$ in [12]):

$$
\Delta l_{\text {elast.ix }}=\Delta x \times \frac{a_{i}}{l_{i}} \leq\left[\Delta l_{\text {elast. } i}\right],
$$

where $\left[\Delta l_{\text {elast. } i}\right]$ - allowable elongation of each flexible element:

$$
\left[\Delta l_{\text {elast. } i}\right]=\left[R_{i 0}\right] \times \frac{1}{7,854 d_{i}^{2}} \times \frac{l_{i}}{n_{i}} .
$$

Here $7.854=\pi \mathrm{E} 10^{-6} / 4$ is a factor of adjustment for elastic modulus of the securing elements as affected by wire twisting (normally $E=1 \cdot 10^{7}$ for annealed wire) [12, p. 200] $\left(\mathrm{kN} / \mathrm{m}^{2}\right) ;\left[R_{i 0}\right]$ - the permissible value of the force in the $I$ elastic fastening element, determined by table 30 in the [16] depending on the number of $n_{i}$ threads (8 PCs.) and the diameter $d_{i}(6 \mathrm{~mm})$ of the fastening wire, taking into account the tension of the 
preliminary twists of the wire $R 0_{i}=20 \mathrm{\kappa H} \mathrm{[19],} \mathrm{т.} \mathrm{Е.}\left[R_{i 0}\right]=\left[R_{i}\right]+R 0_{i}=24.8+20=$ $44.8(\mathrm{kN})$ and $R 0_{i}=18.6+20=38.6(\mathrm{kN})$ for $n_{i}=6(\mathrm{PCs}$.$) and d_{i}=6(\mathrm{~mm})$.

For the given initial data, the allowable values of the elongation of each fastening element were equal to $(\mathrm{m})$ :

$\left[\Delta l_{\text {elast } .1}\right]=0.029,\left[\Delta l_{\text {elast } .2}\right]=0.022,\left[\Delta l_{\text {elast. } 3}\right]=0.021,\left[\Delta l_{\text {elast } .4}\right]=0.028,\left[\Delta l_{\text {elast } .5}\right]=0.019$, $\left[\Delta l_{\text {elast } 6}\right]=0.041-$ for one direction;

$\left[\Delta l_{\text {elast } .1 p}\right]=0.035,\left[\Delta l_{\text {elast } .2 p}\right]=0.05,\left[\Delta l_{\text {elast. } 3 p}\right]=0.021,\left[\Delta l_{\text {elast } .4 p}\right]=0.028,\left[\Delta l_{\text {elast. } 5 p}\right]=$ $0.026,\left[\Delta l_{\text {elast. } 6 p}\right]=0-$ for other direction.

Table 2 presents the calculated elongations of each flexible fastening elements.

Table 2. Calculated elongation of the securing elements in either direction (m).

\begin{tabular}{|c|c|c|c|c|c|c|c|}
\hline $\begin{array}{c}\text { Designation } \\
\text { of } \\
\text { elongation } \\
\text { of the } \\
\text { securing } \\
\text { elements }\end{array}$ & $\begin{array}{c}\text { Direction } \\
\text { of securing }\end{array}$ & \multicolumn{6}{|c|}{ Elongations in the securing elements (m) } \\
\hline \multirow{2}{*}{$\Delta l_{\text {elast.ix }}$} & $\begin{array}{c}\text { One } \\
\text { direction }\end{array}$ & $\Delta l_{\text {elast. } 1 x}$ & $\Delta l_{\text {elast.2x }}$ & $\Delta l_{\text {elast. } 3 x}$ & $\Delta l_{\text {elast. } . x}$ & $\Delta l_{\text {elast. } 5 x}$ & $\Delta l_{\text {elast. } 6 x}$ \\
\cline { 3 - 8 } & 0.036 & 0.0017 & 0.022 & 0.034 & 0.011 & 0.04 \\
\hline \multirow{2}{*}{$\Delta l_{\text {elast.ixp }}$} & $\begin{array}{c}\text { The other } \\
\text { direction }\end{array}$ & $\Delta l_{\text {elast. } 1 x p}$ & $\Delta l_{\text {elast.2xp }}$ & $\Delta l_{\text {elast.3xp }}$ & $\Delta l_{\text {elast. } 4 x p} \Delta l_{\text {elast. } 5 x p}$ & $\Delta l_{\text {elast. } 6 x p}$ \\
\cline { 3 - 9 } & 0.033 & 0.0025 & 0.041 & 0.041 & 0.035 & 0 \\
\hline
\end{tabular}

9.2. Calculation of the force of each flexible fastening element under the influence of longitudinal forces (see (3.91 a) in [12]):

$$
\begin{gathered}
R_{\text {elast.kx }}=7,854 \times d_{i}^{2} \times \frac{n_{i}}{l_{i}} \times \Delta l_{\text {elast } . \mathrm{x} x} \leq\left[R_{i 0}\right] \\
R_{\text {elast.kp }}=7,854 \times d_{i}^{2} \times \frac{n_{i}}{l_{i}} \times \Delta l_{\text {ielast.xp }} \leq\left[R_{i 0}\right](k=\overline{1,2} ; \quad i=\overline{1,6}) .
\end{gathered}
$$

Table 3 presents the calculated forces of securing elements.

\begin{tabular}{|c|c|c|c|c|c|c|c|}
\hline $\begin{array}{c}\text { Designations } \\
\text { of forces in } \\
\text { the securing }\end{array}$ & $\begin{array}{l}\text { Direction } \\
\text { of securing }\end{array}$ & \multicolumn{6}{|c|}{ Forces in the securing elements $(\mathrm{kN})$} \\
\hline \multirow{2}{*}{$R_{\text {elast.ix }}$} & \multirow[t]{2}{*}{$\begin{array}{c}\text { One } \\
\text { direction }\end{array}$} & $R_{\text {elast } 1 x}$ & $R_{\text {elast } 2 x}$ & $R_{\text {elast } 3 x}$ & $R_{\text {elast } 4 x}$ & $R_{\text {elast. } 5 x}$ & $R_{\text {elast. } 6 x}$ \\
\hline & & 55.3 & 3.5 & 46.25 & 53.3 & 25.6 & 43.1 \\
\hline \multirow{2}{*}{$R_{\text {elast.ixp }}$} & \multirow{2}{*}{$\begin{array}{l}\text { The other } \\
\text { direction }\end{array}$} & $R_{\text {elast } 1 x p}$ & $R_{\text {elast } 2 x p}$ & $R_{\text {elast. } 3 x p}$ & $R_{\text {elast } 4 x p}$ & $R_{\text {elast. } 5 x p}$ & $R_{\text {elast.6xp }}$ \\
\hline & & 41.2 & 22.5 & 55.2 & 51.1 & 59.8 & 0 \\
\hline
\end{tabular}

Table 3. Calculated forces of the securing elements in either direction $(\mathrm{kN})$

The analysis of calculation results shows, for the given initial data of a problem (i.e. at coefficient of longitudinal dynamics of the car of $k_{\text {tol. } x}=1.6$ ) efforts in the first, third and fourth flexible elements of fastening of one direction and in the third, fourth and fifth elements of fastening of other direction exceed admissible value $(24.8+20=44.8(\mathrm{kN}))$. Note that the assumed value of the coefficient of longitudinal dynamics of the car $\left(k_{\text {tol. } x}=\right.$ 1.6) corresponds to shunting collision of the car on hump yard marshalling stations. In this case, additional means of fastening are required to ensure the safety of transportation, safety of the transported cargo and parts of the rolling stock. It is possible to control the process of 
calculation, i.e. to predict in advance the possible case of traffic safety violations. At the same time, if the shape of the load and the useful area of the car allows, it is possible to increase the number of fasteners (nail), otherwise - the number of elastic fastening elements, and sometimes perform both. For example, if instead of the accepted number of nails $n_{\text {bar.x }}=6$ (PCs). change the length of the two parallel spacing blocks 5, nailed to the floor of the car close to the transverse-mounted thrust blocks 6 (see Fig. 1), adopt $n_{\text {nail.x }}=16$ (PCs.), leaving the number of thrust bars $n_{\text {bar. }}=16$ (PCs.), then the shift of the cargo along the car $\Delta x$ will decrease by almost 1.3 times (more precisely, instead of 44 and $49(\mathrm{~mm})$ for both directions, respectively, will be equal to 33 and $36(\mathrm{~mm})$. Similarly, reduced elongation on the first, third and fourth fastening elements (for example, the first element is $\Delta l_{\text {elast } .1}=36(\mathrm{~mm})$ will be $26(\mathrm{~mm})$, the third element $\Delta l_{\text {elast } .3}=22(\mathrm{~mm})$ will be $16(\mathrm{~mm})$, and the fourth element $\Delta l_{\text {elast. } 4}=34(\mathrm{~mm})(25 \mathrm{~mm})$. Accordingly, the stress in the first member of one direction would be equal $R_{\text {elast } 1}=40.8$ instead of $55.3(\mathrm{kN})$, i.e. almost 1.35 times $(55.3 / 40.8=1.35)$ less in the fifth element the other direction will be equal to $R_{\text {elast } 5 p}$ $=44.2$ is $59.8(\mathrm{kN})$, i.e. almost 1.25 times $(59.8 / 42.2=1.35)$ is less than it was in $n_{\text {nail. }}=6$ (PCs.). From here it becomes obvious that if the forces in the fastening elements from the effects of longitudinal forces will be less than the permissible, it will be provided with guaranteed strength in the fastening elements with a margin.

We will make a special reservation that similar calculations on the strength of flexible elements of cargo fastening can be carried out also under the influence of transverse forces of one or the other direction.

\section{Conclusions}

1. The sequence of calculation of efforts in elastic elements of fastening of the load (on the example of the winch LP-152) is given on a new method of calculation of fastening is resulted $[12,18]$.

2. The results of calculating the longitudinal forces perceived by the load securing elements are presented, which allowed to determine the shifts of the cargo along the car, elongation and forces in the flexible fastening elements.

3. The results of calculations are similar to [18] revealed that the assumed value of the coefficient of longitudinal dynamics of the car $\left(k_{d . x}=1.6\right)$ corresponds to shunting collision of the car on hump yard of sorting stations. In this case, additional means of fastening, for example, fasteners (nails) are required to ensure the safety of transportation, safety of the transported cargo and parts of the rolling stock. At the same time, if the shape of the cargo and the useful area of the car allows, it is possible to increase the number of fasteners, otherwise - the number of elastic fastening elements, and sometimes perform both.

4. On particular case of the calculation is proved the possibility of calculating the shift of the load along the car and the forces in the fastening elements. The example of calculation expands the possibility of applying in practice a new method of calculation of fastening elements on the car in the development of schemes of placement and fastening of cargoes of arbitrary geometry [12, 16, 18].

\section{References}

1. N. Anderson, P. Anderson, R. Bylander, S. Sokjer, B. Zether: Equipment for Rational Securing of Cargo on railway Wagons / VINNOVA Report/Rapport VR 2004:05 // http://www.vinnova.se/upload / EPiStorePDF /vr-04-05.pdf. 
2. M. Johanson, P. Andersson. Equipment for Efficient Cargo Securing and Ferry Fastening of Vehicles / NVF-rapporter / Vägverket, 2004 //http://www.nvfnorden.org/lisalib/getfile.aspx?itemid=1593

3. Driver's Handbook Cargo Securement. A Cuide to the North American Cargo Securement Standard /http://www.highwaystarmagazine.com/images /Driver_Handbook.pdf

4. European Best Practice Guidelines on Cargo Securing for Road Transport. 208 http://ec.europa.eu/transport/roadsafety/vehicles/best_practice_guidelines_en.htm

5. J.D. Priddy, R.A. Jones, "Experimental Determination of the Effect of Cargo Variations on Roll Stability,” SAE Technical Paper 01, 3516 (2005).

6. J.R. Billing, "Industry Practices for Loading and Securing Cargo in Vans and other Closed Trucks," Ontario Trucking Association (2006).

7. E. Hildebrand, M. Balsom, F.R. Wilson, Impact of Wind Forces on Heavy Truck Stability," Transportation Research Board Annual Meeting (2006).

8. Load Securement for Palletized Freight in Closed Trucks - From an Enforcement Perspective. dPE, Inc. "The Science of Preventing Freight Damage”. (2007).

9. Vertical cylinder handling and transportation AIGA 038/06. http://www.asiaiga.org.

10. E.N. Timukhina, Transport Problems 3(3), 65-68 (2008).

11. A. Manakov, A. Abramov, A. Ilinykh, V. Aksenov, MATEC Web of Conferences 239, 04001 (2018). DOI: 10.1051/matecconf/201823904001.

12. Kh.T. Turanov, Theoretical mechanics in freight transportation problems: monograph (Teoreticheskaya mekhanika $v$ zadachah gruzovyh perevozok) (Novosibirsk, Nauka, 2009)

13. Design loads for axles of wheel pairs (Raschetye nagruzky dlya osey kolesnych par). Railways of the world 2, 36-40, (2011).http://www.bmvit.gv.at, http://www.oevg.at

14. Kh. Turanov, E. Timukhina, Transport Problems 6(3), 27 - 39 (2011).

15. P.S. Anisimov, World of transport 4, 6-13 (2013).

16. K. Turanov. Science and Technology 5(1), 5-14 (2015). JSSN: 2163-2669. (ID: 104000126) doi: 10.5923/j.scit.2015 0501.02).

17. V. Vorobyov, A. Manakov, I. Repina, MATEC Web of Conferences 239, 08011 (2018). DOI: 10.1051/matecconf/201823908011

18. Kh.T. Turanov, E.D. Pserovskaya, Transport of the Urals 3, 16-23, (2013). DOI: 10.20291/1815-9400-2013-3-16-23.

19. E.N. Timukhina, A.A. Gordiienko, Transport of the Urals, 2(45), 33-37, (2015). DOI: 10.20291/1815-9400-2015-2-32-37. 